\title{
Global Exponential Stability of Almost Periodic Solutions for SICNNs with Continuously Distributed Leakage Delays
}

\author{
Hong Zhang ${ }^{1}$ and Mingquan Yang ${ }^{2}$ \\ ${ }^{1}$ College of Mathematics and Computer Science, Hunan University of Arts and Science, Changde, Hunan 415000, China \\ ${ }^{2}$ Nanhu College, Jiaxing University, Jiaxing, Zhejiang 314001, China \\ Correspondence should be addressed to Mingquan Yang; mingquanyang2008@yahoo.com.cn
}

Received 13 November 2012; Revised 23 January 2013; Accepted 24 January 2013

Academic Editor: Wenchang Sun

Copyright (c) $2013 \mathrm{H}$. Zhang and M. Yang. This is an open access article distributed under the Creative Commons Attribution License, which permits unrestricted use, distribution, and reproduction in any medium, provided the original work is properly cited.

Shunting inhibitory cellular neural networks (SICNNs) are considered with the introduction of continuously distributed delays in the leakage (or forgetting) terms. By using the Lyapunov functional method and differential inequality techniques, some sufficient conditions for the existence and exponential stability of almost periodic solutions are established. Our results complement with some recent ones.

\section{Introduction}

It is well known that a neural network usually has a spatial nature due to the presence of an amount of parallel pathways of a variety of axon sizes and lengths; it is desired to model them by introducing continuously distributed delays over a certain duration of time [1-4]. In particular, shunting inhibitory cellular neural networks (SICNNs) with continuously distributed delays can be described by

$$
\begin{aligned}
x_{i j}^{\prime}(t)= & -a_{i j}(t) x_{i j}(t) \\
& -\sum_{C_{k l} \in N_{r}(i, j)} C_{i j}^{k l}(t)\left(x_{k l}(t-\tau(t))\right) x_{i j}(t) \\
& -\sum_{C_{k l} \in N_{q}(i, j)} B_{i j}^{k l}(t) \\
& \cdot \int_{0}^{\infty} K_{i j}(u) g\left(x_{k l}(t-u)\right) d u x_{i j}(t) \\
& +L_{i j}(t), \quad i=1,2, \ldots, m, j=1,2, \ldots, n,
\end{aligned}
$$

where $C_{i j}$ denotes the cell at the $(i, j)$ position of the lattice. The $r$-neighborhood $N_{r}(i, j)$ of is given as

$$
\begin{gathered}
N_{r}(i, j)=\left\{C_{k l}: \max (|k-i|,|l-j|) \leq r,\right. \\
1 \leq k \leq m, 1 \leq l \leq n\},
\end{gathered}
$$

where $N_{q}(i, j)$ is similarly specified, $x_{i j}$ is the activity of the cell $C_{i j}, L_{i j}(t)$ is the external input to $C_{i j}$, the constant $a_{i j}>0$ represents the passive decay rate of the cell activity, $C_{i j}^{k l}(t)$ and $B_{i j}^{k l}(t)$ are the connection or coupling strengths of postsynaptic activity of the cell transmitted to the cell $C_{i j}$, the activity functions $f(\cdot)$ and $g(\cdot)$ are continuous functions representing the output or firing rate of the cell $C_{k l}$, and $\tau(t) \geq$ 0 corresponds to the transmission delay.

Since SICNNs (1) have been introduced as a new cellular neural networks (CNNs) in Bouzerdout and Pinter in [57], it has been extensively applied in psychophysics, speech, perception, robotics, adaptive pattern recognition, vision, and image processing. Hence, there have been extensive results on the problem of the existence and stability of the equilibrium point, periodic and almost periodic solutions of SICNNs with continuously distributed delays in the literature. We refer the reader to [8-12] and references cited therein. 
As pointed out in Gopalsamy [13], the first term in each of the right side of (1) corresponds to a stabilizing negative feedback of the system which acts instantaneously without time delay; these terms are variously known as "forgettin" or leakage terms (see, e.g., Kosko [14] and Haykin [15]). It is known from the literature on population dynamics and neural networks dynamics (see Gopalsamy [16]) that time delays in the stabilizing negative feedback terms will have a tendency to destabilize a system. Therefore, the authors of [17-21] dealt with the existence and stability of equilibrium and periodic solutions for neuron networks model involving leakage delays. Since leakage delays can have a destabilizing influence on the dynamical behaviors of neural networks and the incorporation of time delays in the leakage terms are usually not easy to handle, it necessary to investigate leakage delay effects on the stability of neural networks. On the other hand, as pointed out in $[22,23]$, periodically varying environment and almost periodically varying environment are foundations for the theory of nature selection. Compared with periodic effects, almost periodic effects are more frequent. Hence, the effects of the almost periodic environment on the evolutionary theory have been the object of intensive analysis by numerous authors, and some of these results can be found in $[8,9,11]$ and references cited therein. However, to the best of our knowledge, few authors have considered the existence and exponential stability of almost periodic solutions of SICNNs with continuously distributed delays in the leakage terms. Motivated by the above discussions, in this present paper, we will consider the following SICNNs with continuously distributed leakage delays:

$$
\begin{aligned}
x_{i j}^{\prime}(t)= & -a_{i j}(t) \int_{0}^{\infty} h_{i j}(s) x_{i j}(t-s) d s \\
& -\sum_{C_{k l} \in N_{r}(i, j)} C_{i j}^{k l}(t) f\left(x_{k l}(t-\tau(t))\right) x_{i j}(t) \\
& -\sum_{C_{k l} \in N_{q}(i, j)} B_{i j}^{k l}(t) \int_{0}^{\infty} K_{i j}(u) g\left(x_{k l}(t-u)\right) d u x_{i j}(t) \\
& +L_{i j}(t),
\end{aligned}
$$

where $i=1,2, \ldots, m, j=1,2, \ldots, n, a_{i j}: R \rightarrow(0+\infty), \tau$ : $R \rightarrow[0+\infty)$, and $L_{i j}, C_{i j}^{k l}, B_{i j}^{k l}: R \rightarrow R$ are almost periodic functions, $\tau(t)$ denotes transmission delay, the leakage delay kernels $h_{i j}:[0, \infty) \rightarrow[0, \infty)$ are continuous and integrable, respectively, and the delay kernels $K_{i j}:[0, \infty) \rightarrow[0, \infty)$ are continuous and integrable.

The main purpose of this paper is to give the conditions for the existence and exponential stability of the almost periodic solutions for system (3). By applying the Lyapunov functional method and differential inequality techniques, we derive some new sufficient conditions ensuring the existence, uniqueness, and exponential stability of the almost periodic solution for system (3), which are new and complement previously known results. Moreover, an example is also provided to illustrate the effectiveness of our results.
Throughout this paper, for $i j \in J:=\{11,12, \ldots, 1 n$, $21,22, \ldots, 2 n, \ldots, m 1, m 2, \ldots, m n\}$, delay kernels $h_{i}(s)$ and $K_{i j}(u)$ are continuous functions, and there exist constants $a_{i j}^{+}$ and $\eta_{i j}^{+}$such that

$$
a_{i j}^{+}=\sup _{t \in R} a_{i j}(t), \quad \eta_{i j}^{+}=\int_{0}^{\infty} s h_{i j}(s) d s .
$$

From the theory of almost periodic functions in [22, 23], it follows that, for any $\epsilon>0$, it is possible to find a real number $l=l(\epsilon)>0$, for any interval with length $l(\epsilon)$, and there exists a number $\delta=\delta(\epsilon)$ in this interval such that

$$
\begin{gathered}
\left|a_{i j}(t+\delta)-a_{i j}(t)\right|<\epsilon, \\
\left|C_{i j}^{k l}(t+\delta)-C_{i j}^{k l}(t)\right|<\epsilon, \\
\left|B_{i j}^{k l}(t+\delta)-B_{i j}^{k l}(t)\right|<\epsilon, \\
\quad|\tau(t+\delta)-\tau(t)|<\epsilon, \\
\left|L_{i j}(t+\delta)-L_{i j}(t)\right|<\epsilon,
\end{gathered}
$$

for all $t \in R, k l, i j \in J$.

We set

$$
\begin{gathered}
\left\{x_{i j}(t)\right\}=\left(x_{11}(t), \ldots, x_{1 n}(t), \ldots, x_{i 1}(t), \ldots, x_{i n}(t), \ldots,\right. \\
\left.x_{m 1}(t), \ldots, x_{m n}(t)\right) \in R^{m \times n} .
\end{gathered}
$$

For any $x(t)=\left\{x_{i j}(t)\right\} \in R^{m \times n}$, we define the norm $\|x(t)\|=$ $\max _{(i, j)}\left\{\left|x_{i j}(t)\right|\right\}$. We also assume that the following conditions $\left(T_{1}\right)$ and $\left(T_{2}\right)$ hold.

$\left(T_{1}\right) f: R \rightarrow R$ and $g: R \rightarrow R$ are nonincreasing functions on $[0,+\infty)$, and there exist constants $M_{f}, M_{g}, \mu_{f}$, and $\mu_{g}$ such that

$$
\begin{aligned}
& |f(u)-f(v)| \leq \mu_{f}|u-v|, \\
& |f(u)| \leq M_{f}, \quad|g(u)-g(v)| \leq \mu_{g}|u-v|, \\
& |g(u)| \leq M_{g},
\end{aligned}
$$

$\forall u, v \in R$.

$\left(T_{2}\right)$ For $i j \in J, \quad 1-a_{i j}^{+} \eta_{i j}^{+}>0$

$$
\begin{aligned}
\delta_{i j}(t)= & \left\{\left[a_{i j}(t) \int_{0}^{\infty} h_{i j}(s) d s\left(1-2 a_{i j}^{+} \eta_{i j}^{+}\right)\right.\right. \\
& \left.-\int_{0}^{\infty} h_{i j}(s)\left|a_{i j}(t)-a_{i j}(t-s)\right| d s\right] \\
& -\sum_{C_{k l} \in N_{r}(i, j)}\left|C_{i j}^{k l}(t)\right| M_{f}
\end{aligned}
$$




$$
\begin{aligned}
& \left.\quad-\sum_{C_{k l} \in N_{q}(i, j)}\left|B_{i j}^{k l}(t)\right| \int_{0}^{\infty}\left|K_{i j}(u)\right| d u M_{g}\right\} \\
& \times \frac{1}{1-a_{i j}^{+} \eta_{i j}^{+}} \\
& >0
\end{aligned}
$$

and there exist positive constants $\eta$ and $\lambda$ such that

$$
\begin{gathered}
\lambda<a_{i j}(t) \int_{0}^{\infty} h_{i j}(s) d s, \\
\int_{0}^{\infty} h_{i j}(s) e^{\lambda s} d s<+\infty, \\
\int_{0}^{\infty}\left|K_{i j}(s)\right| e^{\lambda s} d s<+\infty, \\
-\left[\left(a_{i j}(t) \int_{0}^{\infty} h_{i j}(s) d s-\lambda\right)\left(1-2 a_{i j}^{+} \eta_{i j}^{+}\right)\right. \\
\left.+\int_{0}^{\infty} h_{i j}(s)\left|a_{i j}(t) e^{\lambda s}-a_{i j}(t-s)\right| d s\right] \frac{1}{1-a_{i j}^{+} \eta_{i j}^{+}} \\
+\sum_{C_{k l} \in N_{r}(i, j)}\left|C_{i j}^{k l}(t)\right| \mu_{f} e^{\lambda \tau(t)} \frac{1}{1-a_{k l}^{+} \eta_{k l}^{+}} \frac{1}{1-a_{i j}^{+} \eta_{i j}^{+}}\left(\frac{L_{i j}}{\delta_{i j}}\right)^{+} \\
+\sum_{C_{k l} \in N_{r}(i, j)}\left|C_{i j}^{k l}(t)\right| M_{f} \frac{1}{1-a_{i j}^{+} \eta_{i j}^{+}} \\
+\sum_{C_{k l} \in N_{q}(i, j)}\left|B_{i j}^{k l}(t)\right| \int_{0}^{\infty} e^{\lambda u}\left|K_{i j}(u)\right| d u \\
+\sum_{C_{k l} \in N_{q}(i, j)}\left|B_{i j}^{k l}(t)\right| \int_{0}^{\infty}\left|K_{i j}(u)\right| d u M_{g} \frac{1}{1-a_{i j}^{+} \eta_{i j}^{+}} \\
\quad<-\eta, \quad \frac{1}{1-a_{k l}^{+} \eta_{k l}^{+}} \\
\times \frac{1}{1-a_{i j}^{+} \eta_{i j}^{+}}\left(\frac{L_{i j}}{\delta_{i j}}\right)^{+}
\end{gathered}
$$

where $t \geq 0$, and $\left(L_{i j} / \delta_{i j}\right)^{+}=\sup _{t \in R}\left|L_{i j}(t) / \delta_{i j}(t)\right|$. form

The initial conditions associated with system (3) are of the

$$
x_{i j}(s)=\varphi_{i j}(s), \quad s \in(-\infty, 0], i j \in J,
$$

where $\varphi_{i j}(\cdot)$ denotes real-valued bounded continuous function defined on $(-\infty, 0]$.

Definition 1 (see $[22,23])$. Let $u(t): R \rightarrow R^{m \times n}$ be continuous in $t . u(t)$ is said to be almost periodic on $R$ if, for any $\varepsilon>0$, the set $T(u, \varepsilon)=\{\delta:\|u(t+\delta)-u(t)\|<\varepsilon, \forall t \in R\}$ is relatively dense; that is, for any $\varepsilon>0$, it is possible to find a real number $l=l(\varepsilon)>0$, for any interval with length $l(\varepsilon)$, and there exists a number $\delta=\delta(\varepsilon)$ in this interval such that $\|u(t+\delta)-u(t)\|<\varepsilon$, for all $t \in R$.

The remaining part of this paper is organized as follows. In Section 2, we will derive some new sufficient conditions for checking the existence of bounded solutions. In Section 3, we present some new sufficient conditions for the existence, uniqueness and exponential stability of the positive almost periodic solution of (3). In Section 4, we will give some examples and remarks to illustrate our results obtained in previous sections.

\section{Preliminary Results}

The following lemmas will be useful to prove our main results in Section 3.

Lemma 2. Let $\left(T_{1}\right)$ and $\left(T_{2}\right)$ hold. Suppose that $x(t)=\left\{x_{i j}(t)\right\}$ is a solution of system (3) with initial conditions

$$
\begin{gathered}
x_{i j}(s)=\varphi_{i j}(s), \\
\left|\varphi_{i j}(t)-\int_{0}^{\infty} h_{i j}(s) \int_{t-s}^{t} a_{i j}(\theta) \varphi_{i j}(\theta) d \theta d s\right|<\left(\frac{L_{i j}}{\delta_{i j}}\right)^{+},
\end{gathered}
$$

where $s, t \in(-\infty, 0], i j \in J$. Then

$$
\begin{aligned}
\mid x_{i j}(t) & -\int_{0}^{\infty} h_{i j}(s) \int_{t-s}^{t} a_{i j}(\theta) x_{i j}(\theta) d \theta d s \mid \\
\leq & \left(\frac{L_{i j}}{\delta_{i j}}\right)^{+}, \quad \forall t \geq 0, i j \in J, \\
\left|x_{i j}(t)\right| & \leq \frac{1}{1-a_{i j}^{+} \eta_{i j}^{+}}\left(\frac{L_{i j}}{\delta_{i j}}\right)^{+}, \quad \forall t \geq 0, i j \in J .
\end{aligned}
$$

Proof. Assume, by way of contradiction, that (12) does not hold. Then, there exist $i j \in J, \gamma>\left(L_{i j} / \delta_{i j}\right)^{+}$, and $t_{*}>0$ such that

$$
\left|X_{i j}\left(t_{*}\right)\right|=\gamma, \quad\left|X_{i j}(t)\right|<\gamma, \quad \forall t \in\left(-\infty, t_{*}\right),
$$

where

$$
X_{i j}(t)=x_{i j}(t)-\int_{0}^{\infty} h_{i j}(s) \int_{t-s}^{t} a_{i j}(\theta) x_{i j}(\theta) d \theta d s .
$$

It follows that

$$
\begin{aligned}
& \left|x_{i j}(t)\right| \\
& \leq\left|x_{i j}(t)-\int_{0}^{\infty} h_{i j}(s) \int_{t-s}^{t} a_{i j}(\theta) x_{i j}(\theta) d \theta d s\right| \\
& \quad+\left|\int_{0}^{\infty} h_{i j}(s) \int_{t-s}^{t} a_{i j}(\theta) x_{i j}(\theta) d \theta d s\right| \\
& \leq \gamma+a_{i j}^{+} \eta_{i j}^{+} \sup _{s \in\left(-\infty, t_{*}\right]}\left|x_{i j}(s)\right|, \quad \forall t \in\left(-\infty, t_{*}\right] .
\end{aligned}
$$


Consequently, in view of $(16)$ and the fact $a_{i j}^{+} \eta_{i j}^{+}<1(i j \in J)$, we have

$$
\begin{aligned}
\left|x_{i j}(t)\right| & \leq \sup _{s \in\left(-\infty, t_{*}\right]}\left|x_{i j}(s)\right| \\
& \leq \frac{1}{1-a_{i j}^{+} \eta_{i j}^{+}} \gamma, \quad \forall t \in\left(-\infty, t_{*}\right] .
\end{aligned}
$$

From system (3), we derive

$$
\begin{aligned}
& \frac{d}{d t}\left(x_{i j}(t)-\int_{0}^{\infty} h_{i j}(s) \int_{t-s}^{t} a_{i j}(\theta) x_{i j}(\theta) d \theta d s\right) \\
& =x_{i j}^{\prime}(t)-a_{i j}(t) \int_{0}^{\infty} h_{i j}(s) d s x_{i j}(t) \\
& +\int_{0}^{\infty} h_{i j}(s) a_{i j}(t-s) x_{i j}(t-s) d s \\
& =-a_{i j}(t) \int_{0}^{\infty} h_{i j}(s) d s \\
& \times\left(x_{i j}(t)\right. \\
& \left.-\int_{0}^{\infty} h_{i j}(s) \int_{t-s}^{t} a_{i j}(\theta) x_{i j}(\theta) d \theta d s\right) \\
& -a_{i j}(t) \int_{0}^{\infty} h_{i j}(s) d s \int_{0}^{\infty} h_{i j}(s) \\
& \times \int_{t-s}^{t} a_{i j}(\theta) x_{i j}(\theta) d \theta d s \\
& -\int_{0}^{\infty} h_{i j}(s)\left[a_{i j}(t)-a_{i j}(t-s)\right] x_{i j}(t-s) d s \\
& -\sum_{C_{k l} \in N_{r}(i, j)} C_{i j}^{k l}(t) f\left(x_{k l}(t-\tau(t))\right) x_{i j}(t) \\
& -\sum_{C_{k l} \in N_{q}(i, j)} B_{i j}^{k l}(t) \int_{0}^{\infty} K_{i j}(u) g\left(x_{k l}(t-u)\right) d u x_{i j}(t) \\
& +L_{i j}(t), \quad i j \in J \text {. }
\end{aligned}
$$

Calculating the upper left derivative of $\left|X_{i j}(t)\right|$, together with (14), (17), (18), $\left(T_{1}\right)$, and $\left(T_{2}\right)$, we obtain

$$
\begin{aligned}
0 \leq D^{-} & \left|X_{i j}\left(t_{*}\right)\right| \\
\leq & -a_{i j}\left(t_{*}\right) \int_{0}^{\infty} h_{i j}(s) d s\left|X_{i j}\left(t_{*}\right)\right| \\
+ & \mid-a_{i j}\left(t_{*}\right) \int_{0}^{\infty} h_{i j}(s) d s \int_{0}^{\infty} h_{i j}(s) \\
& \times \int_{t_{*}-s}^{t_{*}} a_{i j}(\theta) x_{i j}(\theta) d \theta d s \\
& \quad-\int_{0}^{\infty} h_{i j}(s)\left[a_{i j}\left(t_{*}\right)-a_{i j}\left(t_{*}-s\right)\right] x_{i j}\left(t_{*}-s\right) d s
\end{aligned}
$$

$$
\begin{aligned}
& -\sum_{C_{k l} \in N_{r}(i, j)} C_{i j}^{k l}\left(t_{*}\right) f\left(x_{k l}\left(t_{*}-\tau\left(t_{*}\right)\right)\right) x_{i j}\left(t_{*}\right) \\
& -\sum_{C_{k l} \in N_{q}(i, j)} B_{i j}^{k l}\left(t_{*}\right) \\
& \times \int_{0}^{\infty} K_{i j}(u) g\left(x_{k l}\left(t_{*}-u\right)\right) d u x_{i j}\left(t_{*}\right) \\
& +L_{i j}\left(t_{*}\right) \\
& \leq-a_{i j}\left(t_{*}\right) \int_{0}^{\infty} h_{i j}(s) d s \gamma+a_{i j}\left(t_{*}\right) \\
& \times \int_{0}^{\infty} h_{i j}(s) d s a_{i j}^{+} \eta_{i j}^{+} \frac{1}{1-a_{i j}^{+} \eta_{i j}^{+}} \gamma \\
& +\int_{0}^{\infty} h_{i j}(s)\left|a_{i j}\left(t_{*}\right)-a_{i j}\left(t_{*}-s\right)\right| d s \frac{\gamma}{1-a_{i j}^{+} \eta_{i j}^{+}} \\
& +\sum_{C_{k l} \in N_{r}(i, j)}\left|C_{i j}^{k l}\left(t_{*}\right)\right| M_{f} \frac{1}{1-a_{i j}^{+} \eta_{i j}^{+}} \gamma \\
& +\sum_{C_{k l} \in N_{q}(i, j)}\left|B_{i j}^{k l}\left(t_{*}\right)\right| \int_{0}^{\infty}\left|K_{i j}(u)\right| d u M_{g} \frac{1}{1-a_{i j}^{+} \eta_{i j}^{+}} \gamma \\
& +\left|L_{i j}\left(t_{*}\right)\right| \\
& =\left\{-\left[a_{i j}\left(t_{*}\right) \int_{0}^{\infty} h_{i j}(s) d s\left(1-2 a_{i j}^{+} \eta_{i j}^{+}\right)\right.\right. \\
& \left.-\int_{0}^{\infty} h_{i j}(s)\left|a_{i j}\left(t_{*}\right)-a_{i j}\left(t_{*}-s\right)\right| d s\right] \\
& +\sum_{C_{k l} \in N_{r}(i, j)}\left|C_{i j}^{k l}\left(t_{*}\right)\right| M_{f} \\
& \left.+\sum_{C_{k l} \in N_{q}(i, j)}\left|B_{i j}^{k l}\left(t_{*}\right)\right| \int_{0}^{\infty}\left|K_{i j}(u)\right| d u M_{g}\right\} \frac{1}{1-a_{i j}^{+} \eta_{i j}^{+}} \gamma \\
& +\left|L_{i j}\left(t_{*}\right)\right| \\
& \leq-\delta_{i j}\left(t_{*}\right)\left[\gamma-\left(\frac{L_{i j}}{\delta_{i j}}\right)^{+}\right] \\
& <0 \text {. }
\end{aligned}
$$

It is a contradiction and shows that (12) holds. Then, using a similar argument as in the proof of (16) and (17), we can show that (13) holds. The proof of Lemma 2 is now completed.

Remark 3. In view of the boundedness of this solution, from the theory of functional differential equations with infinite delay in [21], it follows that the solution of system (3) with initial conditions (11) can be defined on $[0, \infty)$. 
Lemma 4. Suppose that $\left(T_{1}\right)$ and $\left(T_{2}\right)$ hold. Moreover, assume that $x(t)=\left\{x_{i j}(t)\right\}$ is a solution of system (3) with initial function $\varphi_{i j}(\cdot)$ satisfying (11), and $\varphi_{i j}^{\prime}(\cdot)$ is bounded continuous on $(-\infty, 0]$. Then, for any $\epsilon>0$, there exists $l=l(\epsilon)>0$, such that every interval $[\alpha, \alpha+l]$ contains at least one number $\delta$ for which there exists $N>0$ which satisfies

$$
\|x(t+\delta)-x(t)\| \leq \epsilon, \quad \forall t>N
$$

Proof. For $i j \in J$, set

$$
\begin{aligned}
\epsilon_{i j}(\delta, t)= & -\left[a_{i j}(t+\delta)-a_{i j}(t)\right] \\
& \times \int_{0}^{\infty} h_{i j}(s) x_{i j}(t+\delta-s) d s \\
& -\sum_{C_{k l} \in N_{r}(i, j)}\left[C_{i j}^{k l}(t+\delta)-C_{i j}^{k l}(t)\right] \\
& \times\left(f\left(x_{k l}(t-\tau(t+\delta)+\delta)\right) x_{i j}(t+\delta)\right) \\
& -\sum_{C_{k l} \in N_{r}(i, j)} C_{i j}^{k l}(t)\left[f\left(x_{k l}(t-\tau(t+\delta)+\delta)\right)\right. \\
& -\sum_{C_{k l} \in N_{q}(i, j)}\left[B_{i j}^{k l}(t+\delta)-B_{i j}^{k l}(t)\right] \\
& \times \int_{0}^{\infty} K_{i j}(u) g\left(x_{k l}(t+\delta-u)\right) d u x_{i j}(t+\delta) \\
& +\left[x_{i j}(t+\delta)-L_{i j}(t)\right] . \\
& \\
& \\
& \\
& \\
&
\end{aligned}
$$

By Lemma 2, the solution $x(t)=\left\{x_{i j}(t)\right\}$ is bounded and

$$
\left|x_{i j}(t)\right| \leq \frac{1}{1-a_{i j}^{+} \eta_{i j}^{+}}\left(\frac{L_{i j}}{\delta_{i j}}\right)^{+}, \quad \forall t \in[0,+\infty), i j \in J
$$

Thus, the right side of (3) is also bounded, which implies that $x(t)$ is uniformly continuous on $R$. From (5), for any $\epsilon>0$, there exists $l=l(\epsilon)>0$, such that every interval $[\alpha, \alpha+l], \alpha \in$ $R$, contains a $\delta$ for which

$$
\left|\epsilon_{i j}(\delta, t)\right| \leq \frac{1}{2} \eta \min _{\widetilde{i} j \in J}\left\{1-a_{i j}^{+} \eta_{i j}^{+}\right\} \epsilon, \quad \text { where } i j \in J, t \in R \text {. }
$$

Let $N_{0} \geq 0$ be sufficiently large such that $t+\delta \geq 0$, for $t \geq N_{0}$, and denote $u_{i j}(t)=x_{i j}(t+\delta)-x_{i j}(t)$. We obtain

$$
\begin{aligned}
& \frac{d u_{i j}(t)}{d t}=-a_{i j}(t) \int_{0}^{\infty} h_{i j}(s) u_{i j}(t-s) d s \\
& -\sum_{C_{k l} \in N_{r}(i, j)} C_{i j}^{k l}(t)\left(f\left(x_{k l}(t-\tau(t)+\delta)\right)\right. \\
& \left.-f\left(x_{k l}(t-\tau(t))\right)\right) \\
& \times x_{i j}(t+\delta) \\
& -\sum_{C_{k l} \in N_{r}(i, j)} C_{i j}^{k l}(t) f\left(x_{k l}(t-\tau(t))\right) \\
& \cdot\left(x_{i j}(t+\delta)-x_{i j}(t)\right) \\
& -\sum_{C_{k l} \in N_{q}(i, j)} B_{i j}^{k l}(t) \int_{0}^{\infty} K_{i j}(u) \\
& \times\left(g\left(x_{k l}(t+\delta-u)\right)\right. \\
& \left.-g\left(x_{k l}(t-u)\right)\right) d u x_{i j}(t+\delta) \\
& -\sum_{C_{k l} \in N_{q}(i, j)} B_{i j}^{k l}(t) \int_{0}^{\infty} K_{i j}(u) g\left(x_{k l}(t-u)\right) d u \\
& \cdot\left(x_{i j}(t+\delta)-x_{i j}(t)\right)+\epsilon_{i j}(\delta, t), \\
& \forall t \geq N_{0}, i j \in J,
\end{aligned}
$$

which yields

$$
\begin{aligned}
& \frac{d}{d t}\left(e^{\lambda t} u_{i j}(t)-\int_{0}^{\infty} h_{i j}(s) \int_{t-s}^{t} a_{i j}(\theta) e^{\lambda \theta} u_{i j}(\theta) d \theta d s\right) \\
&= \lambda e^{\lambda t} u_{i j}(t)+e^{\lambda t} u_{i j}^{\prime}(t) \\
&-a_{i j}(t) \int_{0}^{\infty} h_{i j}(s) d s e^{\lambda t} u_{i j}(t) \\
&+\int_{0}^{\infty} h_{i j}(s) a_{i j}(t-s) e^{\lambda(t-s)} u_{i j}(t-s) d s \\
&=-\left(a_{i j}(t) \int_{0}^{\infty} h_{i j}(s) d s-\lambda\right) \\
& \times\left(e^{\lambda t} u_{i j}(t)-\int_{0}^{\infty} h_{i j}(s) \int_{t-s}^{t} a_{i j}(\theta) e^{\lambda \theta} u_{i j}(\theta) d \theta d s\right) \\
&-\left(a_{i j}(t) \int_{0}^{\infty} h_{i j}(s) d s-\lambda\right) \int_{0}^{\infty} h_{i j}(s) \\
& \times \int_{t-s}^{t} a_{i j}(\theta) e^{\lambda \theta} u_{i j}(\theta) d \theta d s \\
&-\int_{0}^{\infty} h_{i j}(s)\left[a_{i j}(t) e^{\lambda s}-a_{i j}(t-s)\right] \\
& \times e^{\lambda(t-s)} u_{i j}(t-s) d s
\end{aligned}
$$


6

Abstract and Applied Analysis

$$
\begin{aligned}
& +e^{\lambda t}\left\{-\sum_{C_{k l} \in N_{r}(i, j)} C_{i j}^{k l}(t)\right. \\
& \times\left(f\left(x_{k l}(t-\tau(t)+\delta)\right)\right. \\
& \left.-f\left(x_{k l}(t-\tau(t))\right)\right) x_{i j}(t+\delta) \\
& -\sum_{C_{k l} \in N_{r}(i, j)} C_{i j}^{k l}(t) f\left(x_{k l}(t-\tau(t))\right) \\
& \cdot\left(x_{i j}(t+\delta)-x_{i j}(t)\right) \\
& -\sum_{C_{k l} \in N_{q}(i, j)} B_{i j}^{k l}(t) \int_{0}^{\infty} K_{i j}(u) \\
& \times\left(g\left(x_{k l}(t+\delta-u)\right)\right. \\
& \left.-g\left(x_{k l}(t-u)\right)\right) d u x_{i j}(t+\delta) \\
& -\sum_{C_{k l} \in N_{q}(i, j)} B_{i j}^{k l}(t) \int_{0}^{\infty} K_{i j}(u) g\left(x_{k l}(t-u)\right) d u \\
& \left.\cdot\left(x_{i j}(t+\delta)-x_{i j}(t)\right)+\epsilon_{i j}(\delta, t)\right\}, \\
& \forall t \geq N_{0}, \quad i j \in J .
\end{aligned}
$$

Set

$$
U(t)=\left\{U_{i j}(t)\right\}
$$

where

$$
\begin{aligned}
U_{i j}(t)= & e^{\lambda t} u_{i j}(t) \\
& -\int_{0}^{\infty} h_{i j}(s) \int_{t-s}^{t} a_{i j}(\theta) e^{\lambda \theta} u_{i j}(\theta) d \theta d s, \quad i j \in J .
\end{aligned}
$$

Let $(i j)_{t}$ be such an index that

$$
\left|U_{(i j)_{t}}(t)\right|=\|U(t)\| .
$$

Calculating the upper left derivative of $\left|U_{(i j)_{s}}(s)\right|$ along (25), we have

$$
\begin{aligned}
&\left.D^{-}\left(\left|U_{(i j)_{s}}(s)\right|\right)\right|_{s=t} \\
& \leq-\left(a_{(i j)_{t}}(t) \int_{0}^{\infty} h_{(i j)_{t}}(s) d s-\lambda\right)\left|U_{(i j)_{t}}(t)\right| \\
&+ \mid-\left(a_{(i j)_{t}}(t) \int_{0}^{\infty} h_{(i j)_{t}}(s) d s-\lambda\right) \int_{0}^{\infty} h_{(i j)_{t}}(s) \\
& \times \int_{t-s}^{t} a_{(i j)_{t}}(\theta) e^{\lambda \theta} u_{(i j)_{t}}(\theta) d \theta d s \\
&-\int_{0}^{\infty} h_{(i j)_{t}}(s)\left[a_{(i j)_{t}}(t) e^{\lambda s}-a_{(i j)_{t}}(t-s)\right]
\end{aligned}
$$

$$
\begin{aligned}
& \times e^{\lambda(t-s)} u_{(i j)_{t}}(t-s) d s \\
& +e^{\lambda t}\left\{-\sum_{C_{k l} \in N_{r}(i, j)_{t}} C_{(i j)_{t}}^{k l}(t)\right. \\
& \times\left(f\left(x_{k l}(t-\tau(t)+\delta)\right)\right. \\
& \left.-f\left(x_{k l}(t-\tau(t))\right)\right) \\
& \times x_{(i j)_{t}}(t+\delta) \\
& -\sum_{C_{k l} \in N_{r}(i, j)_{t}} C_{(i j)_{t}}^{k l}(t) f\left(x_{k l}(t-\tau(t))\right) \\
& \cdot\left(x_{(i j)_{t}}(t+\delta)-x_{(i j)_{t}}(t)\right) \\
& -\sum_{C_{k l} \in N_{q}(i, j)_{t}} B_{(i j)_{t}}^{k l}(t) \\
& \times \int_{0}^{\infty} K_{(i j)_{t}}(u) \\
& \times\left(g\left(x_{k l}(t+\delta-u)\right)\right. \\
& \left.-g\left(x_{k l}(t-u)\right)\right) d u x_{(i j)_{t}}(t+\delta) \\
& -\sum_{C_{k l} \in N_{q}(i, j)_{t}} B_{(i j)_{t}}^{k l}(t) \\
& \times \int_{0}^{\infty} K_{(i j)_{t}}(u) g\left(x_{k l}(t-u)\right) d u
\end{aligned}
$$

$$
\left.\cdot\left(x_{(i j)_{t}}(t+\delta)-x_{(i j)_{t}}(t)\right)+\epsilon_{(i j)_{t}}(\delta, t)\right\} \mid \text {. }
$$

Let

$$
M(t)=\sup _{s \leq t}\{\|U(s)\|\} .
$$

It is obvious that $\|U(t)\| \leq M(t)$, and $M(t)$ is nondecreasing. In particular,

$$
\begin{aligned}
& e^{\lambda \rho}\left|u_{i j}(\rho)\right| \\
& \leq\left|e^{\lambda \rho} u_{i j}(\rho)-\int_{0}^{\infty} h_{i j}(s) \int_{\rho-s}^{\rho} a_{i j}(\theta) e^{\lambda \theta} u_{i j}(\theta) d \theta d s\right| \\
& \quad+\left|\int_{0}^{\infty} h_{i j}(s) \int_{\rho-s}^{\rho} a_{i j}(\theta) e^{\lambda \theta} u_{i j}(\theta) d \theta d s\right| \\
& \leq M(t) \\
& \quad+a_{i j}^{+} \eta_{i j}^{+} \sup _{\theta \in(-\infty, t]} e^{\lambda \theta}\left|u_{i j}(\theta)\right|, \quad \forall t \geq \rho, i j \in J .
\end{aligned}
$$


Consequently, in view of (31) and the fact $a_{i j}^{+} \eta_{i j}^{+}<1(i j \in J)$, we have

$$
\begin{aligned}
e^{\lambda s}\left|u_{i j}(s)\right| & \leq \sup _{\theta \in(-\infty, t]} e^{\lambda \theta}\left|u_{i j}(\theta)\right| \\
& \leq \frac{M(t)}{1-a_{i j}^{+} \eta_{i j}^{+}}, \quad \text { where } s \in(-\infty, t], i j \in J .
\end{aligned}
$$

Now, we consider two cases.

Case (i). If

$$
M(t)>\|U(t)\|, \quad \forall t \geq N_{0},
$$

then, we claim that

$$
M(t) \equiv M\left(N_{0}\right) \text { is a constant, } \quad \forall t \geq N_{0} .
$$

Assume, by way of contradiction, that (34) does not hold. Then, there exists $t_{1}>N_{0}$, such that $M\left(t_{1}\right)>M\left(N_{0}\right)$, since

$$
\|U(t)\| \leq M\left(N_{0}\right), \quad \forall t \leq N_{0} .
$$

There must exist $\beta \in\left(N_{0}, t_{1}\right)$ such that

$$
\|U(\beta)\|=M\left(t_{1}\right) \geq M(\beta),
$$

which contradicts (33). This contradiction implies that (34) holds. It follows from (32) that there exists $t_{2}>N_{0}$ such that

$$
\begin{aligned}
\|u(t)\| & =\max _{i j \in J}\left|u_{i j}(t)\right| \\
& \leq \max _{i j \in J} \frac{e^{-\lambda t} M(t)}{1-a_{i j}^{+} \eta_{i j}^{+}} \\
& =\max _{i j \in J} \frac{e^{-\lambda t} M\left(N_{0}\right)}{1-a_{i j}^{+} \eta_{i j}^{+}} \\
& <\epsilon, \quad \forall t \geq t_{2} .
\end{aligned}
$$

Case (ii). If there is such a point $t_{0} \geq N_{0}$ that $M\left(t_{0}\right)=\left\|U\left(t_{0}\right)\right\|$, then, in view of (8), (22), (23), (29), (32), $\left(T_{1}\right)$, and $\left(T_{2}\right)$, we get

$$
\begin{aligned}
0 \leq & \left.D^{-}\left(\left|U_{(i j)_{s}}(s)\right|\right)\right|_{s=t_{0}} \\
\leq & -\left(a_{(i j)_{t_{0}}}\left(t_{0}\right) \int_{0}^{\infty} h_{(i j)_{t_{0}}}(s) d s-\lambda\right)\left|U_{(i j)_{t_{0}}}\left(t_{0}\right)\right| \\
& +\left(a_{(i j)_{t_{0}}}\left(t_{0}\right) \int_{0}^{\infty} h_{(i j)_{t_{0}}}(s) d s-\lambda\right) \int_{0}^{\infty} h_{(i j)_{t_{0}}}(s) \\
& \times \int_{t_{0}-s}^{t_{0}} a_{\left(i j_{t_{0}}\right.}^{+} e^{\lambda \theta}\left|u_{(i j)_{t_{0}}}(\theta)\right| d \theta d s \\
& +\int_{0}^{\infty} h_{(i j)_{t_{0}}}(s)\left|a_{(i j)_{t_{0}}}\left(t_{0}\right) e^{\lambda s}-a_{(i j)_{t_{0}}}\left(t_{0}-s\right)\right| \\
& \times e^{\lambda\left(t_{0}-s\right)}\left|u_{(i j)_{t_{0}}}\left(t_{0}-s\right)\right| d s
\end{aligned}
$$

$$
\begin{aligned}
& +\sum_{C_{k l} \in N_{r}(i, j)_{t_{0}}}\left|C_{(i j)_{t_{0}}}^{k l}\left(t_{0}\right)\right| e^{\lambda t_{0}} \\
& \times\left|f\left(x_{k l}\left(t_{0}-\tau\left(t_{0}\right)+\delta\right)\right)-f\left(x_{k l}\left(t_{0}-\tau\left(t_{0}\right)\right)\right)\right| \\
& \times\left|x_{(i j)_{t_{0}}}\left(t_{0}+\delta\right)\right| \\
& +\sum_{C_{k l} \in N_{r}(i, j)_{t_{0}}}\left|C_{(i j)_{t_{0}}}^{k l}\left(t_{0}\right)\right|\left|f\left(x_{k l}\left(t_{0}-\tau\left(t_{0}\right)\right)\right)\right| \\
& \cdot e^{\lambda t_{0}}\left|x_{(i j)_{t_{0}}}\left(t_{0}+\delta\right)-x_{(i j)_{t_{0}}}\left(t_{0}\right)\right| \\
& +\sum_{C_{k l} \in N_{q}(i, j)_{t_{0}}}\left|B_{(i j)_{t_{0}}}^{k l}\left(t_{0}\right)\right| \\
& \times \int_{0}^{\infty}\left|K_{(i j)_{t_{0}}}(u)\right| \\
& \times e^{\lambda t_{0}}\left|g\left(x_{k l}\left(t_{0}+\delta-u\right)\right)-g\left(x_{k l}\left(t_{0}-u\right)\right)\right| d u \\
& \times\left|x_{(i j)_{t_{0}}}\left(t_{0}+\delta\right)\right| \\
& +\sum_{C_{k l} \in N_{q}(i, j)_{t_{0}}}\left|B_{(i j)_{t_{0}}}^{k l}\left(t_{0}\right)\right| \\
& \times \int_{0}^{\infty}\left|K_{(i j)_{t_{0}}}(u)\right| \operatorname{g}\left(x_{k l}\left(t_{0}-u\right)\right) \mid d u \\
& \cdot e^{\lambda t_{0}}\left|x_{(i j)_{t_{0}}}\left(t_{0}+\delta\right)-x_{(i j)_{t_{0}}}\left(t_{0}\right)\right| \\
& +\left|\epsilon_{(i j)_{t_{0}}}\left(\delta, t_{0}\right)\right| e^{\lambda t_{0}} \\
& \leq-\left(a_{(i j)_{t_{0}}}\left(t_{0}\right) \int_{0}^{\infty} h_{(i j)_{t_{0}}}(s) d s-\lambda\right) M\left(t_{0}\right) \\
& +\left(a_{(i j)_{t_{0}}}\left(t_{0}\right) \int_{0}^{\infty} h_{(i j)_{t_{0}}}(s) d s-\lambda\right) a_{(i j)_{t_{0}}}^{+} \eta_{(i j)_{t_{0}}}^{+} \\
& \times \frac{M\left(t_{0}\right)}{1-a_{(i j)_{0}}^{+} \eta_{(i j)_{t_{0}}}^{+}} \\
& +\int_{0}^{\infty} h_{(i j)_{t_{0}}}(s)\left|a_{(i j)_{t_{0}}}\left(t_{0}\right) e^{\lambda s}-a_{(i j)_{t_{0}}}\left(t_{0}-s\right)\right| d s \\
& \times \frac{M\left(t_{0}\right)}{1-a_{(i j)_{0}}^{+} \eta_{(i j)_{t_{0}}}^{+}} \\
& +\sum_{C_{k l} \in N_{r}(i, j)_{t_{0}}}\left|C_{(i j)_{t_{0}}}^{k l}\left(t_{0}\right)\right| \\
& \times \mu_{f} e^{\lambda \tau\left(t_{0}\right)} e^{\lambda\left(t_{0}-\tau\left(t_{0}\right)\right)}\left|u_{k l}\left(t_{0}-\tau\left(t_{0}\right)\right)\right| \\
& \times \frac{1}{1-a_{(i j)_{t_{0}}}^{+} \eta_{(i j)_{t_{0}}}^{+}}\left(\frac{L_{(i j)_{t_{0}}}}{\delta_{(i j)_{t_{0}}}}\right)^{+} \\
& +\sum_{C_{k l} \in N_{r}(i, j)_{t_{0}}}\left|C_{(i j)_{t_{0}}}^{k l}\left(t_{0}\right)\right| M_{f} e^{\lambda t_{0}}\left|u_{(i j)_{t_{0}}}\left(t_{0}\right)\right|
\end{aligned}
$$




$$
\begin{aligned}
& +\sum_{C_{k l} \in N_{q}(i, j)_{t_{0}}}\left|B_{(i j)_{t_{0}}}^{k l}\left(t_{0}\right)\right| \\
& \times \int_{0}^{\infty} e^{\lambda u}\left|K_{(i j)_{t_{0}}}(u)\right| \mu_{g} e^{\lambda\left(t_{0}-u\right)}\left|u_{k l}\left(t_{0}-u\right)\right| d u \\
& \times \frac{1}{1-a_{(i j)_{0}}^{+} \eta_{(i j)_{0}}^{+}}\left(\frac{L_{(i j)_{t_{0}}}}{\delta_{(i j)_{t_{0}}}}\right)^{+} \\
& +\sum_{C_{k l} \in N_{q}(i, j)_{t_{0}}}\left|B_{(i j)_{t_{0}}}^{k l}\left(t_{0}\right)\right| \\
& \times \int_{0}^{\infty}\left|K_{(i j)_{t_{0}}}(u)\right| d u M_{g} e^{\lambda t_{0}}\left|u_{(i j)_{t_{0}}}\left(t_{0}\right)\right| \\
& +\left|\epsilon_{(i j)_{t_{0}}}\left(\delta, t_{0}\right)\right| e^{\lambda t_{0}} \\
& \leq\left\{-\left[\left(a_{(i j)_{t_{0}}}\left(t_{0}\right) \int_{0}^{\infty} h_{(i j)_{t_{0}}}(s) d s-\lambda\right)\right.\right. \\
& \times\left(1-2 a_{(i j)_{t_{0}}}^{+} \eta_{(i j)_{t_{0}}}^{+}\right) \\
& \left.-\int_{0}^{\infty} h_{(i j)_{t_{0}}}(s)\left|a_{(i j)_{t_{0}}}\left(t_{0}\right) e^{\lambda s}-a_{(i j)_{t_{0}}}\left(t_{0}-s\right)\right| d s\right] \\
& \times \frac{1}{1-a_{(i j)_{0}}^{+} \eta_{(i j)_{t_{0}}}^{+}} \\
& +\sum_{C_{k l} \in N_{r}(i, j) t_{t_{0}}}\left|C_{(i j)_{t_{0}}}^{k l}\left(t_{0}\right)\right| \mu_{f} e^{\lambda \tau\left(t_{0}\right)} \\
& \times \frac{1}{1-a_{k l}^{+} \eta_{k l}^{+}} \frac{1}{1-a_{(i j)_{0}}^{+} \eta_{(i j)_{t_{0}}}^{+}}\left(\frac{L_{(i j)_{t_{0}}}}{\delta_{(i j)_{t_{0}}}}\right)^{+} \\
& +\sum_{C_{k l} \in N_{r}(i, j)_{t_{0}}}\left|C_{(i j)_{t_{0}}}^{k l}\left(t_{0}\right)\right| M_{f} \frac{1}{1-a_{(i j)_{t_{0}}}^{+} \eta_{(i j)_{t_{0}}}^{+}} \\
& +\sum_{C_{k l} \in N_{q}(i, j)_{t_{0}}}\left|B_{(i j)_{t_{0}}}^{k l}\left(t_{0}\right)\right| \int_{0}^{\infty} e^{\lambda u}\left|K_{(i j)_{t_{0}}}(u)\right| d u \\
& \times \mu_{g} \frac{1}{1-a_{k l}^{+} \eta_{k l}^{+}} \frac{1}{1-a_{(i j)_{t_{0}}}^{+} \eta_{(i j)_{t_{0}}}^{+}}\left(\frac{L_{(i j)_{t_{0}}}}{\delta_{(i j)_{t_{0}}}}\right)^{+} \\
& +\sum_{C_{k l} \in N_{q}(i, j)_{t_{0}}}\left|B_{(i j)_{t_{0}}}^{k l}\left(t_{0}\right)\right| \\
& \left.\times \int_{0}^{\infty}\left|K_{(i)_{t_{0}}}(u)\right| d u M_{g} \frac{1}{1-a_{(i j)_{t_{0}}}^{+} \eta_{(i j)_{t_{0}}}^{+}}\right\} M\left(t_{0}\right) \\
& +\eta \min _{i j \in J}\left\{1-a_{i j}^{+} \eta_{i j}^{+}\right\} \epsilon e^{\lambda t_{0}} \\
& <-\eta M\left(t_{0}\right)+\eta \min _{i j \in J}\left\{1-a_{i j}^{+} \eta_{i j}^{+}\right\} \epsilon e^{\lambda t_{0}},
\end{aligned}
$$

which yields that

$$
\begin{gathered}
\left\|U\left(t_{0}\right)\right\|=M\left(t_{0}\right)<\min _{i j \in J}\left\{1-a_{i j}^{+} \eta_{i j}^{+}\right\} \epsilon e^{\lambda t_{0}}, \\
\left\|u\left(t_{0}\right)\right\| \leq \max _{i j \in J} \frac{e^{-\lambda t_{0}} M\left(t_{0}\right)}{1-a_{i j}^{+} \eta_{i j}^{+}}<\epsilon .
\end{gathered}
$$

For any $t>t_{0}$, by the same approach used in the proof of (39), we have

$$
\begin{aligned}
& \|U(t)\|=M(t)<\min _{i j \in J}\left\{1-a_{i j}^{+} \eta_{i j}^{+}\right\} \epsilon e^{\lambda t}, \\
& \|u(t)\|<\epsilon,
\end{aligned}
$$

$$
\text { if } M(t)=\|U(t)\| .
$$

On the other hand, if $M(t)>\|U(t)\|$ and $t>t_{0}$, we can choose $t_{0} \leq t_{3}<t$ such that

$$
M\left(t_{3}\right)=\left\|U\left(t_{3}\right)\right\|, \quad M(s)>\|U(s)\|, \quad \forall s \in\left(t_{3}, t\right],
$$

which, together with (40), yields that

$$
\begin{gathered}
M\left(t_{3}\right)=\left\|U\left(t_{3}\right)\right\|<\min _{i j \in J}\left\{1-a_{i j}^{+} \eta_{i j}^{+}\right\} \epsilon e^{\lambda t_{3}}, \\
\left\|u\left(t_{3}\right)\right\|<\epsilon .
\end{gathered}
$$

Using a similar argument as in the proof of Case (i), we can show that

$$
M(s) \equiv M\left(t_{3}\right) \text { is a constant, } \quad \forall s \in\left(t_{3}, t\right],
$$

which implies that

$$
\begin{aligned}
\|u(t)\| & \leq \max _{i j \in J} \frac{e^{-\lambda t} M(t)}{1-a_{i j}^{+} \eta_{i j}^{+}} \\
& =\max _{i j \in J} \frac{e^{-\lambda t} M\left(t_{3}\right)}{1-a_{i j}^{+} \eta_{i j}^{+}} \\
& <\max _{i j \in J} \frac{e^{-\lambda t} \min _{\bar{i} j \in J}\left\{1-a_{i j}^{+} \eta_{i j}^{+}\right\} \epsilon e^{\lambda t_{3}}}{1-a_{i j}^{+} \eta_{i j}^{+}} \\
& <\epsilon .
\end{aligned}
$$

In summary, there must exist $N>\max \left\{t_{0}, N_{0}, t_{2}\right\}$ such that $\|u(t)\| \leq \epsilon$ holds, for all $t>N$. The proof of Lemma 4 is now complete.

\section{Main Results}

In this section, we establish some results for the existence, uniqueness, and exponential stability of the almost periodic solution of (3).

Theorem 5. Suppose that $\left(T_{1}\right)$ and $\left(T_{2}\right)$ are satisfied. Then system (3) has exactly one almost periodic solution $Z^{*}(t)$. Moreover, $Z^{*}(t)$ is globally exponentially stable. 
Proof. Let $v(t)=\left\{v_{i j}(t)\right\}$ be a solution of system (3) with initial function $\varphi_{i j}^{v}(\cdot)$ satisfying $(11)$, and $\left(\varphi_{i j}^{v}(\cdot)\right)^{\prime}$ is bounded continuous on $(-\infty, 0]$.

Set

$$
\begin{aligned}
\epsilon_{i j, k}(t)= & -\left[a_{i j}\left(t+t_{k}\right)-a_{i j}(t)\right] \\
& \times \int_{0}^{\infty} h_{i j}(s) v_{i j}\left(t+t_{k}-s\right) d s \\
& -\sum_{C_{k l} \in N_{r}(i, j)}\left[C_{i j}^{k l}\left(t+t_{k}\right)-C_{i j}^{k l}(t)\right] \\
& \times\left(f\left(x_{k l}\left(t-\tau\left(t+t_{k}\right)+t_{k}\right)\right)\right) x_{i j}\left(t+t_{k}\right) \\
& -\sum_{C_{k l} \in N_{r}(i, j)} C_{i j}^{k l}(t) \\
& +\sum_{C_{k l} \in N_{q}(i, j)}\left[B_{i j}^{k l}\left(t+t_{k}\right)-B_{i j}^{k l}(t)\right] \\
& \times \int_{0}^{\infty} K_{i j}(u) g\left(x_{k l}\left(t-\tau\left(t+t_{k}\right)+t_{k}\right)\right) \\
& \\
& \left.\left.\times x_{i j}\left(t+t_{k}\right)-t_{k}\left(t-\tau(t)+t_{k}\right)\right)\right] \\
&
\end{aligned}
$$

where $\left\{t_{k}\right\}$ is any sequence of real numbers. By Lemma 2, the solution $v(t)$ is bounded and

$$
\left|v_{i j}(t)\right| \leq \frac{1}{1-a_{i j}^{+} \eta_{i j}^{+}}\left(\frac{L_{i j}}{\delta_{i j}}\right)^{+}, \quad \forall t \in R, i j \in J,
$$

which implies that the right side of (3) is also bounded, and $v^{\prime}(t)$ is a bounded function on $R$. Thus, $v(t)$ is uniformly continuous on $R$. Then, from the almost periodicity of $a_{i j}, \tau, C_{i j}^{k l}$, and $B_{i j}^{k l}$, we can select a sequence $\left\{t_{k}\right\} \rightarrow+\infty$ such that

$$
\begin{gathered}
\left|a_{i j}\left(t+t_{k}\right)-a_{i j}(t)\right| \leq \frac{1}{k}, \\
\left|C_{i j}^{k l}\left(t+t_{k}\right)-C_{i j}^{k l}(t)\right| \leq \frac{1}{k}, \\
\left|B_{i j}^{k l}\left(t+t_{k}\right)-B_{i j}^{k l}(t)\right| \leq \frac{1}{k}, \\
\left|\tau\left(t+t_{k}\right)-\tau(t)\right| \leq \frac{1}{k}, \\
\left|\epsilon_{i j, k}(t)\right| \leq \frac{1}{k},
\end{gathered}
$$

for all $i j, k l \in J, t \in R$.

Since $\left\{v\left(t+t_{k}\right)\right\}_{k=1}^{+\infty}$ is uniformly bounded and equiuniformly continuous, by the Arzala-Ascoli Lemma and diagonal selection principle, we can choose a subsequence $\left\{t_{k_{j}}\right\}$ of $\left\{t_{k}\right\}$, such that $v\left(t+t_{k_{j}}\right)$ (for convenience, we still denote by $\left.v\left(t+t_{k}\right)\right)$ uniformly converges to a continuous function $Z^{*}(t)=\left\{x_{i j}^{*}(t)\right\}$ on any compact set of $R$, and

$$
\left|x_{i j}^{*}(t)\right| \leq \frac{1}{1-a_{i j}^{+} \eta_{i j}^{+}}\left(\frac{L_{i j}}{\delta_{i j}}\right)^{+}, \quad \forall t \in R, i j \in J .
$$

Now, we prove that $Z^{*}(t)$ is a solution of (3). In fact, for any $t>0$ and $\Delta t \in R$, from (47), we have

$$
\begin{aligned}
& x_{i j}^{*}(t+\Delta t)-x_{i j}^{*}(t) \\
& =\lim _{k \rightarrow+\infty}\left[v_{i j}\left(t+\Delta t+t_{k}\right)-v_{i j}\left(t+t_{k}\right)\right] \\
& =\lim _{k \rightarrow+\infty} \int_{t}^{t+\Delta t}\left\{-a_{i j}\left(\mu+t_{k}\right)\right. \\
& \times \int_{0}^{\infty} h_{i j}(s) v_{i j}\left(\mu+t_{k}-s\right) d s \\
& -\sum_{C_{k l} \in N_{r}(i, j)} C_{i j}^{k l}\left(\mu+t_{k}\right) \\
& \times f\left(v_{k l}\left(\mu+t_{k}-\tau\left(\mu+t_{k}\right)\right)\right) \\
& \times v_{i j}\left(\mu+t_{k}\right) \\
& -\sum_{C_{k l} \in N_{q}(i, j)} B_{i j}^{k l}\left(\mu+t_{k}\right) \\
& \times \int_{0}^{\infty} K_{i j}(u) g \\
& \times\left(v_{k l}\left(\mu+t_{k}-u\right)\right) d u \\
& \times v_{i j}\left(\mu+t_{k}\right) \\
& \left.+L_{i j}\left(\mu+t_{k}\right)\right\} d \mu \\
& =\lim _{k \rightarrow+\infty} \int_{t}^{t+\Delta t}\left\{-a_{i j}(\mu) \int_{0}^{\infty} h_{i j}(s) v_{i j}\left(\mu+t_{k}-s\right) d s\right. \\
& -\sum_{C_{k l} \in N_{r}(i, j)} C_{i j}^{k l}(\mu) f \\
& \times\left(v_{k l}\left(\mu-\tau(\mu)+t_{k}\right)\right) \\
& \times v_{i j}\left(\mu+t_{k}\right) \\
& -\sum_{C_{k l} \in N_{q}(i, j)} B_{i j}^{k l}(\mu) \\
& \times \int_{0}^{\infty} K_{i j}(u) g\left(v_{k l}\left(\mu+t_{k}-u\right)\right) \\
& \times d u v_{i j}\left(\mu+t_{k}\right) \\
& \left.+L_{i j}(\mu)+\epsilon_{i j, k}(\mu)\right\} d \mu
\end{aligned}
$$




$$
\begin{aligned}
& =\int_{t}^{t+\Delta t}\left\{-a_{i j}(\mu) \int_{0}^{\infty} h_{i j}(s) x_{i j}^{*}(\mu-s) d s\right. \\
& -\sum_{C_{k l} \in N_{r}(i, j)} C_{i j}^{k l}(\mu) f\left(x_{k l}^{*}(\mu-\tau(\mu))\right) x_{i j}^{*}(\mu) \\
& -\sum_{C_{k l} \in N_{q}(i, j)} B_{i j}^{k l}(\mu) \\
& \times \int_{0}^{\infty} K_{i j}(u) g\left(x_{k l}^{*}(\mu-u)\right) d u x_{i j}^{*}(\mu) \\
& \left.+L_{i j}(\mu)\right\} d \mu \\
& +\lim _{k \rightarrow+\infty} \int_{t}^{t+\Delta t} \epsilon_{i j, k}(\mu) d \mu \\
& =\int_{t}^{t+\Delta t}\left\{-a_{i j}(\mu) \int_{0}^{\infty} h_{i j}(s) x_{i j}^{*}(\mu-s) d s\right. \\
& \text { - } \sum_{C_{k l} \in N_{r}(i, j)} C_{i j}^{k l}(\mu) f\left(x_{k l}^{*}(\mu-\tau(\mu))\right) x_{i j}^{*}(\mu) \\
& -\sum_{C_{k l} \in N_{q}(i, j)} B_{i j}^{k l}(\mu) \\
& \times \int_{0}^{\infty} K_{i j}(u) g\left(x_{k l}^{*}(\mu-u)\right) d u x_{i j}^{*}(\mu) \\
& \left.+L_{i j}(\mu)\right\} d \mu,
\end{aligned}
$$

which implies that

$$
\begin{aligned}
\frac{d}{d t}\left\{x_{i j}^{*}(t)\right\}= & -a_{i j}(t) \int_{0}^{\infty} h_{i j}(s) x_{i j}^{*}(t-s) d s \\
& -\sum_{C_{k l} \in N_{r}(i, j)} C_{i j}^{k l}(t) f\left(x_{k l}^{*}(t-\tau(t))\right) x_{i j}^{*}(t) \\
& -\sum_{C_{k l} \in N_{q}(i, j)} B_{i j}^{k l}(t) \\
& \times \int_{0}^{\infty} K_{i j}(u) g\left(x_{k l}^{*}(t-u)\right) d u x_{i j}^{*}(t) \\
& +L_{i j}(t), \quad i j \in J .
\end{aligned}
$$

Therefore, $Z^{*}(t)$ is a solution of (3).

Secondly, we prove that $Z^{*}(t)$ is an almost periodic solution of (3). From Lemma 4, for any $\varepsilon>0$, there exists $l=l(\varepsilon)>0$, such that every interval $[\alpha, \alpha+l]$ contains at least one number $\delta$ for which there exists $N>0$ which satisfies

$$
\left|v_{i j}(t+\delta)-v_{i j}(t)\right| \leq \varepsilon, \quad \forall t>N, i j \in J
$$

Then, for any fixed $s \in R$, we can find a sufficiently large positive integer $N_{1}>N$ such that, for any $k>N_{1}$,

$$
\begin{gathered}
s+t_{k}>N, \\
\left|v_{i j}\left(s+t_{k}+\delta\right)-v_{i j}\left(s+t_{k}\right)\right| \leq \varepsilon, \quad i j \in J .
\end{gathered}
$$

Let $k \rightarrow+\infty$; we obtain

$$
\left|x_{i j}^{*}(s+\delta)-x_{i j}^{*}(s)\right| \leq \varepsilon, \quad i j \in J,
$$

which implies that $Z^{*}(t)$ is an almost periodic solution of (3).

Finally, we prove that $Z^{*}(t)$ is globally exponentially stable.

Let $Z^{*}(t)=\left\{x_{i j}^{*}(t)\right\}$ be the positive almost periodic solution of system (3) with initial value $\varphi^{*}=\left\{\varphi_{i j}^{*}(t)\right\}$ and $Z(t)=\left\{x_{i j}(t)\right\}$ an arbitrary solution of system (3) with initial value $\varphi=\left\{\varphi_{i j}(t)\right\}$, and set $y(t)=\left\{y_{i j}(t)\right\}=\left\{x_{i j}(t)-x_{i j}^{*}(t)\right\}=$ $Z(t)-Z^{*}(t)$. Then

$$
\begin{aligned}
y_{i j}^{\prime}(t)= & -a_{i j}(t) \int_{0}^{\infty} h_{i j}(s) y_{i j}(t-s) d s \\
& -\sum_{C_{k l} \in N_{r}(i, j)} C_{i j}^{k l}(t) \\
\times & {\left[f\left(x_{k l}(t-\tau(t))\right) x_{i j}(t)\right.} \\
& \left.-f\left(x_{k l}^{*}(t-\tau(t))\right) x_{i j}^{*}(t)\right] \\
-\sum_{C_{k l} \in N_{q}(i, j)} B_{i j}^{k l}(t) & \\
\times & {\left[\int_{0}^{\infty} K_{i j}(u) g\left(x_{k l}(t-u)\right) d u x_{i j}(t)\right.} \\
& \left.-\int_{0}^{\infty} K_{i j}(u) g\left(x_{k l}^{*}(t-u)\right) d u x_{i j}^{*}(t)\right],
\end{aligned}
$$

which yields

$$
\begin{aligned}
\frac{d}{d t}\left(e^{\lambda t} y_{i j}(t)-\int_{0}^{\infty} h_{i j}(s) \int_{t-s}^{t} a_{i j}(\theta) e^{\lambda \theta} y_{i j}(\theta) d \theta d s\right) \\
=\lambda e^{\lambda t} y_{i j}(t)+e^{\lambda t} y_{i j}^{\prime}(t) \\
\quad-a_{i j}(t) \int_{0}^{\infty} h_{i j}(s) d s e^{\lambda t} y_{i j}(t) \\
\quad+\int_{0}^{\infty} h_{i j}(s) a_{i j}(t-s) e^{\lambda(t-s)} y_{i j}(t-s) d s \\
=-\left(a_{i j}(t) \int_{0}^{\infty} h_{i j}(s) d s-\lambda\right) \\
\quad \times\left(e^{\lambda t} y_{i j}(t)-\int_{0}^{\infty} h_{i j}(s) \int_{t-s}^{t} a_{i j}(\theta) e^{\lambda \theta} y_{i j}(\theta) d \theta d s\right)
\end{aligned}
$$




$$
\begin{aligned}
& -\left(a_{i j}(t) \int_{0}^{\infty} h_{i j}(s) d s-\lambda\right) \int_{0}^{\infty} h_{i j}(s) \\
& \times \int_{t-s}^{t} a_{i j}(\theta) e^{\lambda \theta} y_{i j}(\theta) d \theta d s \\
& -\int_{0}^{\infty} h_{i j}(s)\left[a_{i j}(t) e^{\lambda s}-a_{i j}(t-s)\right] \\
& \times e^{\lambda(t-s)} y_{i j}(t-s) d s \\
& +e^{\lambda t}\left\{-\sum_{C_{k l} \in N_{r}(i, j)} C_{i j}^{k l}(t)\right. \\
& \times\left[f\left(x_{k l}(t-\tau(t))\right)-f\left(x_{k l}^{*}(t-\tau(t))\right)\right] x_{i j}(t) \\
& -\sum_{C_{k l} \in N_{r}(i, j)} C_{i j}^{k l}(t) f\left(x_{k l}^{*}(t-\tau(t))\right) \\
& \times\left[x_{i j}(t)-x_{i j}^{*}(t)\right] \\
& -\sum_{C_{k l} \in N_{q}(i, j)} B_{i j}^{k l}(t) \int_{0}^{\infty} K_{i j}(u) \\
& \times\left[g\left(x_{k l}(t-u)\right)-g\left(x_{k l}^{*}(t-u)\right)\right] d u x_{i j}(t) \\
& -\sum_{C_{k l} \in N_{q}(i, j)} B_{i j}^{k l}(t) \int_{0}^{\infty} K_{i j}(u) g \\
& \left.\times\left(x_{k l}^{*}(t-u)\right) d u\left[x_{i j}(t)-x_{i j}^{*}(t)\right]\right\},
\end{aligned}
$$

where $i j \in J$.

Let

$$
\begin{aligned}
Y_{i j}(t)= & \mid e^{\lambda t} y_{i j}(t)-\int_{0}^{\infty} h_{i j}(s) \\
& \int_{t-s}^{t} a_{i j}(\theta) e^{\lambda \theta} y_{i j}(\theta) d \theta d s \mid, \quad i j \in J .
\end{aligned}
$$

We define a positive constant $M$ as follows:

$$
M=\max _{i j \in J}\left\{\sup _{s \in(-\infty, 0]} Y_{i j}(s)\right\} .
$$

Let $K$ be a positive number such that

$$
Y_{i j}(t) \leq M<M+1=K, \quad \forall t \in(-\infty, 0], i j \in J .
$$

We claim that

$$
Y_{i j}(t)<K, \quad \forall t>0, i=1,2, \ldots, n .
$$

Otherwise, there must exist $i j \in J$ and $\varsigma>0$ such that

$$
Y_{i j}(\varsigma)=K, \quad Y_{\widetilde{i} j}(t)<K, \quad \forall t \in(-\infty, \varsigma), \widetilde{i} j \in J .
$$

It follows that

$$
\begin{aligned}
& e^{\lambda t}\left|y_{i j}(t)\right| \leq \mid e^{\lambda t} y_{i j}(t)-\int_{0}^{\infty} h_{i j}(s) \\
& \times \int_{t-s}^{t} a_{\tilde{i} j}(\theta) e^{\lambda \theta} y_{i j}(\theta) d \theta d s \mid \\
&+\left|\int_{0}^{\infty} h_{\tilde{i} j}(s) \int_{t-s}^{t} a_{\tilde{i j}}(\theta) e^{\lambda \theta} y_{\tilde{i} j}(\theta) d \theta d s\right| \\
& \leq K+a_{i j}^{+} \eta_{\tilde{i j}}^{+} \sup _{s \in(-\infty, \varsigma]} e^{\lambda s}\left|y_{i j}(s)\right|, \\
& \forall t \in(-\infty, \varsigma], \widetilde{i} j \in J .
\end{aligned}
$$

Consequently, in view of (61) and the fact $a_{i j}^{+} \eta_{i j}^{+}<1(\widetilde{i j} \in J)$, we have

$$
\begin{aligned}
e^{\lambda t}\left|y_{i j}(t)\right| & \leq \sup _{s \in(-\infty, \varsigma]} e^{\lambda s}\left|y_{i j}(s)\right| \\
& \leq \frac{K}{1-a_{i j}^{+} \eta_{i j}^{+}}, \quad \forall t \in(-\infty, \varsigma], \widetilde{i} j \in J .
\end{aligned}
$$

Calculating the upper left derivative of $Y_{i j}(t)$, together with (13), (55), (60), (62), $\left(T_{1}\right)$, and $\left(T_{2}\right)$, we obtain

$$
\begin{aligned}
0 \leq D^{-} Y_{i j}(\varsigma) & \\
\leq- & \left(a_{i j}(\varsigma) \int_{0}^{\infty} h_{i j}(s) d s-\lambda\right) Y_{i j}(\varsigma) \\
+ & -\left(a_{i j}(\varsigma) \int_{0}^{\infty} h_{i j}(s) d s-\lambda\right) \\
& \times \int_{0}^{\infty} h_{i j}(s) \int_{\varsigma-s}^{\varsigma} a_{i j}(\theta) e^{\lambda \theta} y_{i j}(\theta) d \theta d s \\
& -\int_{0}^{\infty} h_{i j}(s)\left[a_{i j}(\varsigma) e^{\lambda s}-a_{i j}(\varsigma-s)\right] \\
& \times e^{\lambda(\varsigma-s)} y_{i j}(\varsigma-s) d s \\
& +e^{\lambda \varsigma}\left\{\begin{array}{c}
-\sum_{C_{k l} \in N_{r}(i, j)} C_{i j}^{k l}(\varsigma) \\
\times\left[f\left(x_{k l}(\varsigma-\tau(\varsigma))\right)-f\left(x_{k l}^{*}(\varsigma-\tau(\varsigma))\right)\right] \\
\times x_{i j}(\varsigma) \\
\sum_{k l} \in N_{r}(i, j)
\end{array} C_{i j}^{k l}(\varsigma) f\left(x_{k l}^{*}(\varsigma-\tau(\varsigma))\right)\right.
\end{aligned}
$$




$$
\begin{aligned}
& \times\left[x_{i j}(\varsigma)-x_{i j}^{*}(\varsigma)\right] \\
& -\sum_{C_{k l} \in N_{q}(i, j)} B_{i j}^{k l}(\varsigma) \\
& \times \int_{0}^{\infty} K_{i j}(u) \\
& \times\left[g\left(x_{k l}(\varsigma-u)\right)-g\left(x_{k l}^{*}(\varsigma-u)\right)\right] \\
& \times d u x_{i j}(\varsigma) \\
& -\sum_{C_{k l} \in N_{q}(i, j)} B_{i j}^{k l}(\varsigma) \\
& \times \int_{0}^{\infty} K_{i j}(u) g\left(x_{k l}^{*}(\varsigma-u)\right) d u \\
& \left.\times\left[x_{i j}(\varsigma)-x_{i j}^{*}(\varsigma)\right]\right\} \mid \\
& \leq-\left(a_{i j}(\varsigma) \int_{0}^{\infty} h_{i j}(s) d s-\lambda\right) Y_{i j}(\varsigma) \\
& +\left(a_{i j}(\varsigma) \int_{0}^{\infty} h_{i j}(s) d s-\lambda\right) \frac{K}{1-a_{i j}^{+} \eta_{i j}^{+}} a_{i j}^{+} \eta_{i j}^{+} \\
& +\int_{0}^{\infty} h_{i j}(s)\left|a_{i j}(\varsigma) e^{\lambda s}-a_{i j}(\varsigma-s)\right| \\
& \times e^{\lambda(\varsigma-s)}\left|y_{i j}(\varsigma-s)\right| d s \\
& +\sum_{C_{k l} \in N_{r}(i, j)}\left|C_{i j}^{k l}(\varsigma)\right| \mu_{f} e^{\lambda \tau(\varsigma)} e^{\lambda(\varsigma-\tau(\varsigma))} \\
& \times\left|y_{k l}(\varsigma-\tau(\varsigma))\right|\left|x_{i j}(\varsigma)\right| \\
& +\sum_{C_{k l} \in N_{r}(i, j)}\left|C_{i j}^{k l}(\varsigma)\right| M_{f} e^{\lambda \varsigma}\left|y_{i j}(\varsigma)\right| \\
& +\sum_{C_{k l} \in N_{q}(i, j)}\left|B_{i j}^{k l}(\varsigma)\right| \int_{0}^{\infty} e^{\lambda u} \\
& \times\left|K_{i j}(u)\right| \mu_{g} e^{\lambda(\varsigma-u)}\left|y_{k l}(\varsigma-u)\right| d u\left|x_{i j}(\varsigma)\right| \\
& +\sum_{C_{k l} \in N_{q}(i, j)}\left|B_{i j}^{k l}(\varsigma)\right| \\
& \times \int_{0}^{\infty}\left|K_{i j}(u)\right| d u \\
& \times M_{g} e^{\lambda \varsigma}\left|y_{i j}(\varsigma)\right| \\
& \leq\left\{-\left[\left(a_{i j}(\varsigma) \int_{0}^{\infty} h_{i j}(s) d s-\lambda\right)\left(1-2 a_{i j}^{+} \eta_{i j}^{+}\right)\right.\right. \\
& \left.-\int_{0}^{\infty} h_{i j}(s)\left|a_{i j}(\varsigma) e^{\lambda s}-a_{i j}(\varsigma-s)\right| d s\right]
\end{aligned}
$$

$$
\begin{aligned}
& \times \frac{1}{1-a_{i j}^{+} \eta_{i j}^{+}} \\
& +\sum_{C_{k l} \in N_{r}(i, j)}\left|C_{i j}^{k l}(\varsigma)\right| \mu_{f} e^{\lambda \tau(\varsigma)} \\
& \times \frac{1}{1-a_{k l}^{+} \eta_{k l}^{+}} \frac{1}{1-a_{i j}^{+} \eta_{i j}^{+}}\left(\frac{L_{i j}}{\delta_{i j}}\right)^{+} \\
& +\sum_{C_{k l} \in N_{r}(i, j)}\left|C_{i j}^{k l}(\varsigma)\right| M_{f} \frac{1}{1-a_{i j}^{+} \eta_{i j}^{+}} \\
& +\sum_{C_{k l} \in N_{q}(i, j)}\left|B_{i j}^{k l}(\varsigma)\right| \int_{0}^{\infty} e^{\lambda u}\left|K_{i j}(u)\right| d u \\
& \times \mu_{g} \frac{1}{1-a_{k l}^{+} \eta_{k l}^{+}} \frac{1}{1-a_{i j}^{+} \eta_{i j}^{+}}\left(\frac{L_{i j}}{\delta_{i j}}\right)^{+} \\
& +\sum_{C_{k l} \in N_{q}(i, j)}\left|B_{i j}^{k l}(\varsigma)\right| \int_{0}^{\infty}\left|K_{i j}(u)\right| d u \\
& \left.\times M_{g} \frac{1}{1-a_{i j}^{+} \eta_{i j}^{+}}\right\} K \\
& <-\eta K
\end{aligned}
$$$$
<0 \text {, }
$$

which is a contradiction and implies that (59) holds.

Consequently, using a similar argument as in (61)-(62), we know that

$$
\left|y_{i j}(t)\right| e^{\lambda t} \leq \frac{K}{1-a_{i j}^{+} \eta_{i j}^{+}}, \quad \forall t \in R, i j \in J .
$$

This completes the proof.

\section{An Example}

In this section, we give an example to demonstrate the results obtained in the previous sections.

Example 6. Consider the following SICNNs with continuously distributed delays in the leakage terms:

$$
\begin{aligned}
\frac{d x_{i j}}{d t}= & -a_{i j}(t) \int_{0}^{\infty} h_{i j}(s) x_{i j}(t-s) d s \\
& -\sum_{C_{k l} \in N_{r}(i, j)} C_{i j}^{k l}(t) f\left(x_{k l}(t-\tau(t))\right) x_{i j}(t) \\
& -\sum_{C_{k l} \in N_{q}(i, j)} B_{i j}^{k l}(t) \int_{0}^{\infty} K_{i j}(u) g\left(x_{k l}(t-u)\right) d u x_{i j}(t) \\
& +L_{i j}(t), \quad i, j=1,2,3,
\end{aligned}
$$




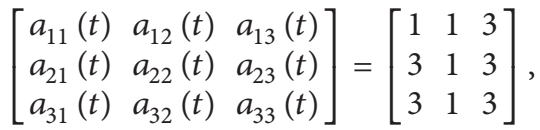

$$
\begin{aligned}
& {\left[\begin{array}{lll}
B_{11}(t) & B_{12}(t) & B_{13}(t) \\
B_{21}(t) & B_{22}(t) & B_{23}(t) \\
B_{31}(t) & B_{32}(t) & B_{33}(t)
\end{array}\right]=\left[\begin{array}{lll}
C_{11}(t) & C_{12}(t) & C_{13}(t) \\
C_{21}(t) & C_{22}(t) & C_{23}(t) \\
C_{31}(t) & C_{32}(t) & C_{33}(t)
\end{array}\right]} \\
& =\left[\begin{array}{ccc}
0.1 & 0.2 & 0.1 \\
0.2 & 0 & 0.2 \\
0.1 & 0.2 & 0.1
\end{array}\right] \\
& {\left[\begin{array}{lll}
L_{11}(t) & L_{12}(t) & L_{13}(t) \\
L_{21}(t) & L_{22}(t) & L_{23}(t) \\
L_{31}(t) & L_{32}(t) & L_{33}(t)
\end{array}\right]} \\
& =0.05 \\
& \times\left[\begin{array}{ccc}
0.7+0.24 \sin ^{2} \sqrt{2} t & 0.41+0.5 \cos ^{2} t & 0.74+0.2 \sin ^{2} t \\
0.61+0.2 \cos ^{2} t & 0.67+0.2 \sin ^{2} t & 0.75+0.2 \sin ^{2} t \\
0.59+0.4 \cos ^{4} t & 0.5+0.41 \sin ^{2} t & 0.76+0.2 \cos ^{2} t
\end{array}\right] .
\end{aligned}
$$

Set $\lambda=0.001, \eta=0.05, r=q=1, h_{i j}(s)=(1 / 5) e^{-s^{2}}, K_{i j}(u)=$ $|\sin u| e^{-u}, i=1,2$, and $3 . j=1,2$, and 3 , and $f(x)=$ $g(x)=(1 / 4000)(|x-1|-|x+1|), \tau(t)=(1 / 100)$ $\sin ^{2} t$; clearly, $M_{f}=M_{g}=0.0005, \mu_{f}=\mu_{g}=0.0005$, $\sum_{C_{k l} \in N_{1}(1,1)} C_{11}^{k l}=\sum_{C_{k l} \in N_{1}(1,1)} B_{11}^{k l}=0.5, \sum_{C_{k l} \in N_{1}(1,2)} C_{12}^{k l}=$ $\sum_{C_{k l} \in N_{1}(1,2)} B_{12}^{k l}=0.8, \sum_{C_{k l} \in N_{1}(1,3)} C_{13}^{k l}=\sum_{C_{k l} \in N_{1}(1,3)} B_{13}^{k l}=$ $0.5, \sum_{C_{k l} \in N_{1}(2,1)} C_{21}^{k l}=\sum_{C_{k l} \in N_{1}(2,1)} B_{21}^{k l}=0.8, \sum_{C_{k l} \in N_{1}(2,2)} C_{22}^{k l}=$ $\sum_{C_{k l} \in N_{1}(2,2)} B_{22}^{k l}=1.2, \sum_{C_{k l} \in N_{1}(2,3)} C_{23}^{k l}=\sum_{C_{k l} \in N_{1}(2,3)} B_{23}^{k l}=$ $0.8, \sum_{C_{k l} \in N_{1}(3,1)} C_{31}^{k l}=\sum_{C_{k l} \in N_{1}(3,1)} B_{31}^{k l}=0.5, \sum_{C_{k l} \in N_{1}(3,2)} C_{32}^{k l}=$ $\sum_{C_{k l} \in N_{1}(3,2)} B_{32}^{k l}=0.8, \sum_{C_{k l} \in N_{1}(3,3)} C_{33}^{k l}=\sum_{C_{k l} \in N_{1}(3,3)} B_{33}^{k l}=0.5$, $1 \leq a_{i j}^{+} \leq 3, \eta_{i j}^{+}=0.1, a_{i j}^{+} \eta_{i j}^{+} \leq 0.3<1$, and $i j \in J=$ $\{11,12,13,21,22,23,31,32,33\}$.

Consider,

$$
\begin{gathered}
\min _{i j \in J} \delta_{i j}(t) \\
=\min _{i j \in J}\left\{\left[a_{i j}(t) \int_{0}^{\infty} h_{i j}(s) d s\left(1-2 a_{i j}^{+} \eta_{i j}^{+}\right)\right.\right. \\
\left.-\int_{0}^{\infty} h_{i j}(s)\left|a_{i j}(t)-a_{i j}(t-s)\right| d s\right] \\
-\sum_{C_{k l} \in N_{r}(i, j)}\left|C_{i j}^{k l}(t)\right| M_{f} \\
\left.-\sum_{C_{k l} \in N_{q}(i, j)}\left|B_{i j}^{k l}(t)\right| \int_{0}^{\infty}\left|K_{i j}(u)\right| d u M_{g}\right\} \\
\times \frac{1}{1-a_{i j}^{+} \eta_{i j}^{+}}
\end{gathered}
$$$$
>0.0715>0, \quad \forall t \geq 0 \text {. }
$$

$$
\begin{aligned}
& \max _{i j \in J}\left\{-\left[\left(a_{i j}(t) \int_{0}^{\infty} h_{i j}(s) d s-\lambda\right)\left(1-2 a_{i j}^{+} \eta_{i j}^{+}\right)\right.\right. \\
& \left.-\int_{0}^{\infty} h_{i j}(s)\left|a_{i j}(t) e^{\lambda s}-a_{i j}(t-s)\right| d s\right] \\
& \times \frac{1}{1-a_{i j}^{+} \eta_{i j}^{+}} \\
& +\sum_{C_{k l} \in N_{r}(i, j)}\left|C_{i j}^{k l}(t)\right| \mu_{f} e^{\lambda \tau(t)} \\
& \times \frac{1}{1-a_{k l}^{+} \eta_{k l}^{+}} \frac{1}{1-a_{i j}^{+} \eta_{i j}^{+}}\left(\frac{L_{i j}}{\delta_{i j}}\right)^{+} \\
& +\sum_{C_{k l} \in N_{r}(i, j)}\left|C_{i j}^{k l}(t)\right| M_{f} \frac{1}{1-a_{i j}^{+} \eta_{i j}^{+}} \\
& +\sum_{C_{k l} \in N_{q}(i, j)}\left|B_{i j}^{k l}(t)\right| \int_{0}^{\infty} e^{\lambda u}\left|K_{i j}(u)\right| d u \\
& \times \mu_{g} \frac{1}{1-a_{k l}^{+} \eta_{k l}^{+}} \frac{1}{1-a_{i j}^{+} \eta_{i j}^{+}}\left(\frac{L_{i j}}{\delta_{i j}}\right)^{+} \\
& +\sum_{C_{k l} \in N_{q}(i, j)}\left|B_{i j}^{k l}(t)\right| \int_{0}^{\infty}\left|K_{i j}(u)\right| d u \\
& \left.\times M_{g} \frac{1}{1-a_{i j}^{+} \eta_{i j}^{+}}\right\} \\
& <-0.0556<-0.05=-\eta, \quad \forall t \geq 0 .
\end{aligned}
$$

It follows that system (65) satisfies all the conditions in Theorem 5. Hence, system (65) has exactly one almost periodic solution. Moreover, the almost periodic solution is globally exponentially stable.

Remark 7. Since [1-11] only dealt with SICNNs without leakage delays, [12-21] give no opinions about the problem of almost periodic solutions for SICNNs with leakage delays. One can observe that all the results in these literature and the references therein can not be applicable to prove the existence and exponential stability of almost periodic solutions for SICNNs (65).

\section{Acknowledgments}

The authors would like to express the sincere appreciation to the reviewers for their helpful comments in improving the presentation and quality of the paper. In particular, the authors expresses the sincere gratitude to Professor Bingwen Liu for the helpful discussion when this work is carried out. This work was supported by the National Natural Science Foundation of China (Grant no. 11201184), the Natural Scientific Research Fund of Hunan Provincial of China (Grant no. 11JJ6006), the Natural Scientific Research Fund 
of Hunan Provincial Education Department of China (Grant nos. 11C0916 and 11C0915), the Natural Scientific Research Fund of Zhejiang Provincial of China (Grants nos. Y6110436 and LY12A01018), and the Natural Scientific Research Fund of Zhejiang Provincial Education Department of China (Grant no. Z201122436).

\section{References}

[1] J. H. Park, "Further result on asymptotic stability criterion of cellular neural networks with time-varying discrete and distributed delays," Applied Mathematics and Computation, vol. 182, no. 2, pp. 1661-1666, 2006.

[2] X. Li, C. Ding, and Q. Zhu, "Synchronization of stochastic perturbed chaotic neural networks with mixed delays," Journal of the Franklin Institute, vol. 347, no. 7, pp. 1266-1280, 2010.

[3] O. M. Kwon and J. H. Park, "Delay-dependent stability for uncertain cellular neural networks with discrete and distribute time-varying delays," Journal of the Franklin Institute, vol. 345, no. 7, pp. 766-778, 2008.

[4] L. Wang, "Stability of Cohen-Grossberg neural networks with distributed delays," Applied Mathematics and Computation, vol. 160, no. 1, pp. 93-110, 2005.

[5] A. Bouzerdoum and R. B. Pinter, "Shunting inhibitory cellular neural networks: derivation and stability analysis," IEEE Transactions on Circuits and Systems. I. Fundamental Theory and Applications, vol. 40, no. 3, pp. 215-221, 1993.

[6] A. Bouzerdoum and R. B. Pinter, "Analysis and analog implementation of directionally sensitive shunting inhibitory cellular neural networks," in Visual Information Processing: From neurons to Chips, vol. 1473 of Proceedings of SPIE, pp. 29-38, 1991.

[7] A. Bouzerdoum and R. B. Pinter, "Nonlinear lateral inhibition applied to motion detection in the fly visual system," in Nonlinear Vision, R. B. Pinter and B. Nabet, Eds., pp. 423-450, CRC Press, Boca Raton, Fla, USA, 1992.

[8] B. Liu and L. Huang, "Existence and stability of almost periodic solutions for shunting inhibitory cellular neural networks with continuously distributed delays," Physics Letters A, vol. 349, pp. 177-186, 2006.

[9] Q. Fan and J. Shao, "Positive almost periodic solutions for shunting inhibitory cellular neural networks with time-varying and continuously distributed delays," Communications in Nonlinear Science and Numerical Simulation, vol. 15, no. 6, pp. 1655-1663, 2010.

[10] Q. Zhou and C. Zhao, "Exponential convergence of shunting inhibitory cellular neural networks with continuously distributed delays," Annales Polonici Mathematici, vol. 98, no. 3, pp. 241-250, 2010.

[11] Q. Zhou, B. Xiao, Y. Yu, and L. Peng, "Existence and exponential stability of almost periodic solutions for shunting inhibitory cellular neural networks with continuously distributed delays," Chaos, Solitons and Fractals, vol. 34, no. 3, pp. 860-866, 2007.

[12] Z. Chen, "A shunting inhibitory cellular neural network with leakage delays and continuously distributed delays of neutral type," Neural Computing and Applications, 2012.

[13] K. Gopalsamy, "Leakage delays in BAM," Journal of Mathematical Analysis and Applications, vol. 325, no. 2, pp. 1117-1132, 2007.

[14] B. Kosko, Neural Networks and Fuzzy Systems, Prentice-Hall, Englewood Cliffs, NJ, USA, 1992.

[15] S. Haykin, Neural Networks, Prentice Hall, New Jersey, NY, USA, 1999.
[16] K. Gopalsamy, Stability and Oscillations in Delay Differential Equations of Population Dynamics, vol. 74 of Mathematics and Its Applications, Kluwer Academic Publishers, Dordrecht, The Netherlands, 1992.

[17] X. Li and J. Cao, "Delay-dependent stability of neural networks of neutral type with time delay in the leakage term," Nonlinearity, vol. 23, no. 7, pp. 1709-1726, 2010.

[18] X. Li, R. Rakkiyappan, and P. Balasubramaniam, "Existence and global stability analysis of equilibrium of fuzzy cellular neural networks with time delay in the leakage term under impulsive perturbations," Journal of the Franklin Institute, vol. 348, no. 2, pp. 135-155, 2011.

[19] P. Balasubramaniam, V. Vembarasan, and R. Rakkiyappan, "Leakage delays in T-S fuzzy cellular neural networks," Neural Processing Letters, vol. 33, pp. 111-136, 2011.

[20] B. Liu, "Global exponential stability for BAM neural networks with time-varying delays in the leakage terms," Nonlinear Analysis. Real World Applications, vol. 14, no. 1, pp. 559-566, 2013.

[21] Z. Chen and M. Yang, "Exponential convergence for HRNNs with continuously distributed delays in the leakage terms," Neural Computing \& Applications, 2012.

[22] A. M. Fink, Almost Periodic Differential Equations, vol. 377 of Lecture Notes in Mathematics, Springer, Berlin, Germany, 1974.

[23] C. Y. He, Almost Periodic Differential Equation, House, Higher Education Publishing, Beijing, China, 1992. 


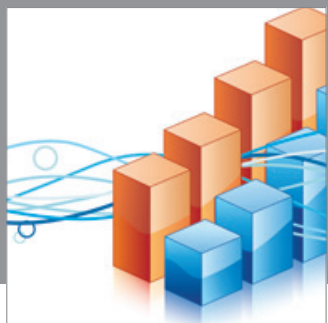

Advances in

Operations Research

mansans

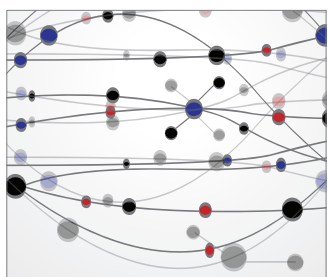

The Scientific World Journal
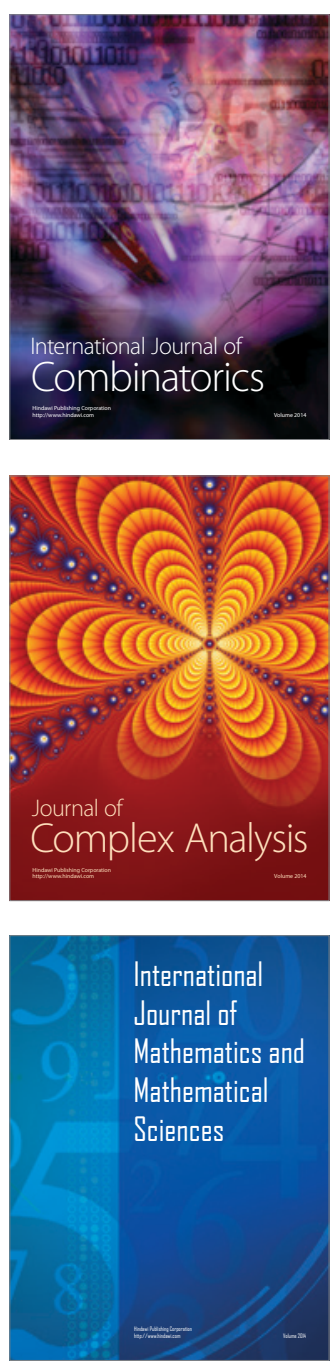
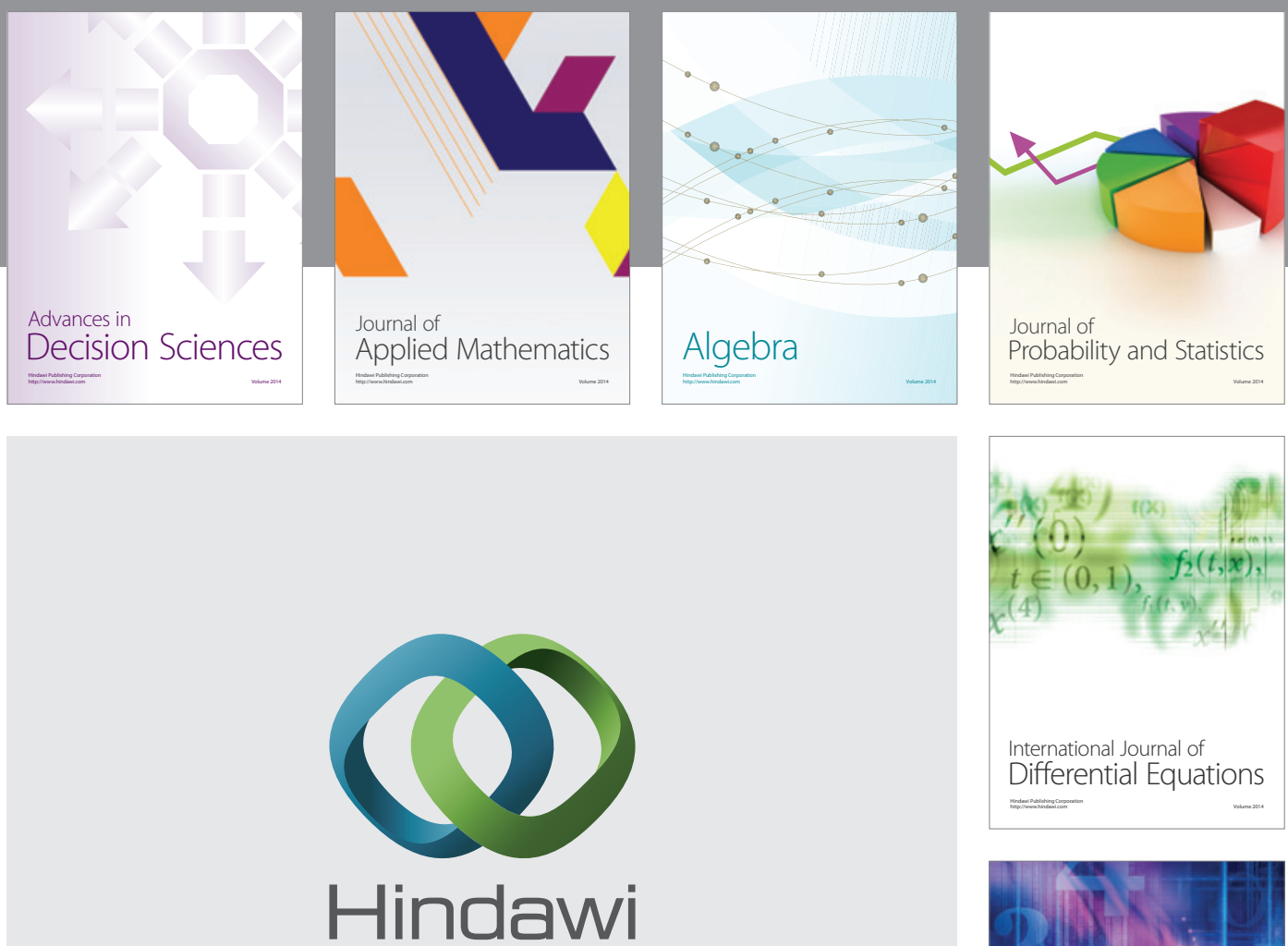

Submit your manuscripts at http://www.hindawi.com
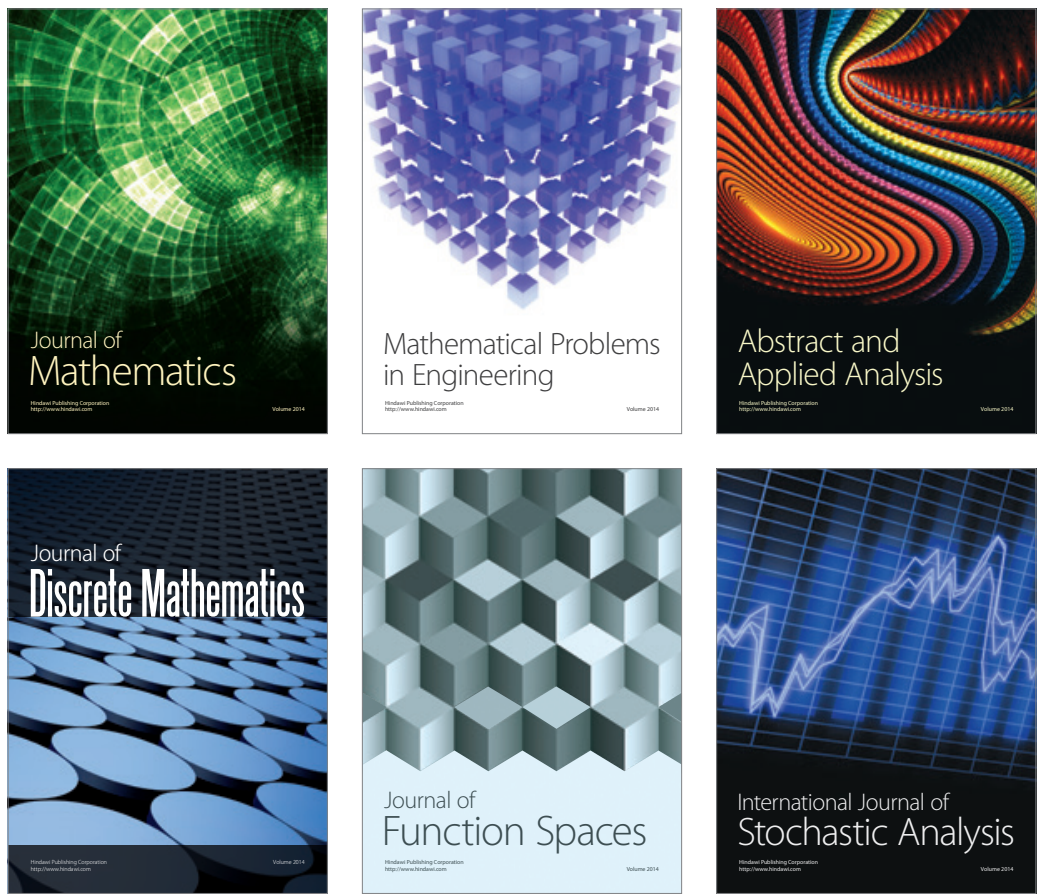

Journal of

Function Spaces

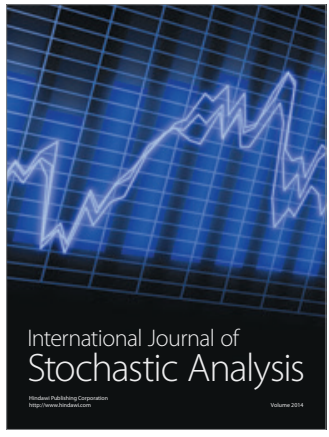

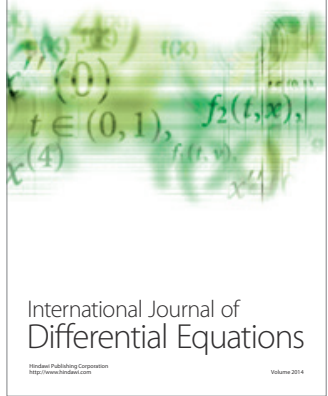
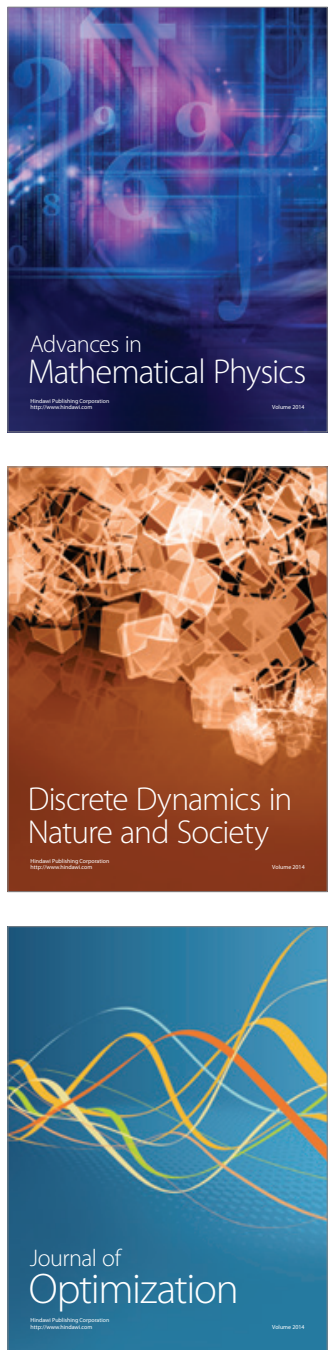